\title{
The Thermodynamic Models of Molecular Chemical Compound Distribution in the Giant Molecular Clouds Medium
}

\author{
Michel Yu. Dolomatov ${ }^{1} \&$ Nadezhda A. Zhuravleva $^{1}$ \\ ${ }^{1}$ Department of Physics, Ufa State Academy of Economics and Service, 145 Chernyshevskiy St., Ufa, Russian \\ Federation \\ Correspondence: Nadezhda A. Zhuravleva, 450078 Department of Physics, Ufa State Academy of Economics and \\ Service, 145 Chernyshevskiy St., Ufa, Russian Federation. Tel: 7-919-158-7048. E-mail: zhuravliova80@mail.ru, \\ dolomatov@gmail.com
}

\author{
Received: September 25, 2012 Accepted: October 11, 2012 Online Published: October 30, 2012 \\ doi:10.5539/apr.v4n4p149 URL: http://dx.doi.org/10.5539/apr.v4n4p149
}

\begin{abstract}
The new equilibrium thermodynamic distribution model of organic and bio-organic substances in giant molecular clouds, including simple organic molecules and amino acids and nucleic acid components was developed. The model basis comprises of the equilibrium composition distribution functions construction by the standard enthalpy of formation and free energy (Helmholtz energy) of formation, which are calculated by means of standard methods. Based on these models the organic molecules resources forecast within the Metagalaxy was performed. The significant amount of biologically active substances existence possibility is indicated: amino acids, nucleobases.
\end{abstract}

Keywords: giant molecular clouds, free energy (Helmholtz energy) of formation, standard enthalpy of formation, amino acids, nucleobases, organic molecules

\section{Introduction}

The organic substances resources assessment in the Universe is a crucial and vital task in connection with the living and nonliving substances evolution study. The radio astronomy data, obtained in recent years (Rudnitskiy, 1999) indicate that the organic substance of cosmic origin is concentrated in the gas molecules interstellar clusters, which are called giant molecular clouds (GMCs).

The first thermodynamic models of molecular substances distribution in interstars media was displayed the in the research (Dolomatov, 2001-2004). These models allowed only for the distribution on heat formation.

Let's consider thermodynamic features of GMC as multi-component systems with the chemical composition chaos (MSCC). On the basis of the existence of more than 165 molecules in the GMC (Molecules in Space, 2012), it can be assumed that in volume of these systems there are areas in which conditions for local thermodynamic equilibrium are created. In addition, it can be noted that the temperature in these areas range from $2-700 \mathrm{~K}$. At higher temperatures under the influence of a hard radiation simple organic molecules dissociate with ions or free radicals formation. Thus, from the fact of sustainable observation of stable molecules in the interstellar medium, it follows that the principle of local equilibrium in some areas of the interstellar medium is performed, and if so, then the laws of equilibrium thermodynamics can be used for quantitative assessment of molecular resources. It is no coincidence; that the basis of many of Physical-chemical calculations in the interstellar medium are accepted the conditions of local equilibrium (Heyer et al., 2009; Vasyunin, 2008; Bains et al., 2006; Sakamoto, 1996; Lee et al., 1996; Maloney, 1986).

\section{Research Methods}

It is unknown at the present time the full range of physical and chemical processes in the GMC, determined by the temperature and concentration gradients, the shock waves, heavy-duty electromagnetic and gravitational fields, etc. That is why, it incorrect to attribute it to the well-defined laboratory conditions and the well-known chemical reactions mechanisms. Therefore, a phenomenological approach is appropriate for the chemical composition distribution in GMCs models (Dolomatov, 2005).

According to the theory, the GMC belongs to the MSCC (Dolomatov, 2005). 
The peculiarity of the MSCC, including the GMCs, presents the possibility of a large number of different nature components existing in the substance elementary volume - from simple molecules to the complex substances. Following the theory (Dolomatov, 2005), we shall distinguish an isolated MSCC is the statistical ensemble consisting of a large number of components - $N$, each of which is characterized by a specific value of the thermodynamic potential or property. Defining the possibility of the existence in a system of $M$ components, with specific thermodynamic potential or properties, which is different from the average system's property. Such a possibility $(W)$ is determined by the binomial (Bernoulli) distribution:

$$
W=C_{N}^{M} \cdot p^{M} \cdot(1-p)^{N-M}
$$

where $C_{N}{ }^{M}$ - binomial coefficient: $N$ by $M ; p=1-1 / z$ the probability of the components micro conditions chemical difference $z$. When $z=>\infty$ the system is constructed from the entirely different components, $p=>1$. When $z=1$ $p=0$ the system consists of a single component, but such a situation is not possible, according to the $2^{\text {nd }}$ thermodynamics law, the system conditions diversity increase takes place. When $p \rightarrow 0$, we obtain the system with the thermodynamic characteristics Poisson nature of distribution (pure substances).

In typical MSCC cases, the possibilities $0<p<1$ are implemented (for example, the hydrocarbon systems). This can be concluded from the Laplace-Moivre theorem. In this case (1) Gaussian distribution of the composition by the components thermodynamic potentials and properties is formed.

The MSCC thermodynamics suggests that the normal composition distribution law by free energies should be observed:

$$
F\left(p_{i}\right)=\frac{1}{\sigma \sqrt{2 \pi}} \exp \left[-\left(\frac{\Delta F-F_{S}}{\sigma \sqrt{2}}\right)^{2}\right]
$$

where $p_{i}$ - probability existing molecules in MSCC with the defined free energy (Helmholtz energy), $\sigma^{2}-$ dispersion, $\Delta F$ - free energy (Helmholtz energy) of $i$-compound, $\Delta \bar{F}$ - average value of all molecular compounds.

In accordance with the MSCC thermodynamics there should be similar organic substances distribution by the standard enthalpy of formation:

$$
H\left(p_{i}\right)=\frac{1}{\sigma \sqrt{2 \pi}} \exp \left[-\left(\frac{\Delta H-\Delta \bar{H}}{\sigma \sqrt{2}}\right)^{2}\right]
$$

where $p_{i}$ - probability existing molecules in MSCC with the defined standard enthalpy of formation, $\sigma^{2}$ dispersion, $\Delta H$ - standard enthalpy of formation of i-compound, $\Delta \bar{H}$ - average value of all molecular compounds standard enthalpy of formation.

The standard enthalpy and free energy (Helmholtz energy) of the compounds are calculated by molecular and spectroscopy data (Landau \& Lifshitz, 1996).

The mistake in the previous study was due to the lack of strict statistical approach in taking into account the small samples of statistics, the unreasonable calculation of algorithms and considering several distribution laws. This finally led to the ambiguity in the statistical estimates.

The purpose of this study is to estimate the molecules resources in the GMC in accordance with the normal distribution law.

\section{Subjects of Research}

The investigation and research objects of the molecules, which exist in the GMC, was proved by using radio spectral methods (Lovas, 1992; Molecules in Space, 2012). The information on 67 molecular organic compounds was analyzed: the oxygen-containing molecules, nitrogen-containing molecules, the methane series and the acetylene hydrocarbons. In addition, taking into account the recent data, the aromatic hydrocarbons, fullerenes, glycine and simple carbohydrates was also investigated (Cami et al., 2010). The organic compounds standard enthalpy of formation at the standard temperature of $298 \mathrm{~K}$ was selected as the properties. In order to study the formation enthalpy, the temperature range from $100 \mathrm{~K}$ to $600 \mathrm{~K}$ was selected, i.e., the temperature range, providing simple organic molecules stable existence in the GMC substance at low density conditions.

Assumptions of the mathematical model of the chemical composition in GMC distribution: 
1) Sizes of the Metagalaxy part where resource material evaluation is carried out is limited by the visibility horizon of modern means of surveillance.

2) In accordance with the modern physics presentation, the universal isotropy and homogeneity is assumed, as well as the GMC formation processes permanence.

3) Despite the fact that in some areas of GMC the star formation takes place and the substance is in the state of the non-equilibrium plasma, in the system there are areas that are in a state of local thermodynamic equilibrium and have temperatures $<1000 \mathrm{~K}$, in which the molecules of organic substances are stable.

4) The phenomenological model: GMC represents a range of similar cybernetic "black boxes" within the physical and chemical conditions complex. They are exposed to perturbation of disturbing environmental conditions. This is why there are a lot of conditions and the probabilities of any substance formation. The specific chemical reactions mechanism is not considered. The input parameters are the properties, the output parameters - is the amount of substance.

5) When the phenomenological distribution models construction the averaged data in accordance with the large numbers effect law, the areas by the GMC are considered with a uniform set of conditions. According to Lyapunov's theorem, the regular composition distribution shall be expected by the properties in case of homogeneous conditions complex, but given the conditions heterogeneity, generating the multi-dimensional distributions and the condensation physical processes (the substance accretion).

6) The carbon content in the universe is constant, i.e., its flow rate is in the process of accretion and star formation is equal to the rate of its reproduction in the fusion process and supernova explosions. It is considered that the explosions of supernovae enrich space with active particles of carbon.

7) This estimate does not include the hidden mass, which according to various assessments, 5 - 10 times exceeds the visible mass.

Based on the above stated, the following model is proposed:

$$
M_{i}=x F\left(p_{i}\right) Q N_{g} M_{o}
$$

where $p_{i}-i$-molecule existence possibility in the GMC,

$F\left(p_{i}\right)$ - the corresponding possibility distribution function,

$Q$ - average number of GMC in the Galaxy,

$N_{g}$ - average number of galaxies in the visible part of Universe,

$M_{o}=2 \cdot 10^{30} \mathrm{~kg}-$ Sun mass,

$x=1$ - the coefficient, taking into account the organic molecules loss during the star formation processes and their reproduction at supernova stars explosions.

In accordance with the estimates, accepted in the astrophysics (Binney \& Merrifield, 1998) $Q=1 \div 10^{6} ; N_{g}=10^{10} ; x$ $=1$.

The calculations algorithm

The calculations were carried out using the following algorithm:

1) The variational series construction and their characteristics determination on the compounds thermodynamic properties (Table 1). 
Table 1. Probability of existence of organic connections with certain free energy (Helmholtz energy) of formation at $100 \mathrm{~K}$ in GMC Distribution

\begin{tabular}{cccc}
\hline \multicolumn{2}{c}{ Variation interval } & $\begin{array}{c}\text { Number of connections in an } \\
\text { interval, } n\end{array}$ & Existence of connections probability, $p$ \\
\hline$-37,64$ & 4,07 & 4 & $-16,79$ \\
4,07 & 45,77 & 7 & 24,92 \\
45,77 & 87,48 & 15 & 66,63 \\
87,48 & 129,19 & 15 & 108,33 \\
129,19 & 170,89 & 13 & 150,04 \\
170,89 & 212,60 & 10 & 191,75 \\
212,60 & 254,31 & 2 & 233,45 \\
254,31 & 296,01 & 1 & 275,16 \\
\hline
\end{tabular}

2) The compounds distribution functions construction and their analysis (Table 2).

Table 2. Regular distribution criteria statistical evaluation

\begin{tabular}{ccc}
\hline \multirow{2}{*}{ Index } & \multicolumn{2}{c}{ Value } \\
\cline { 2 - 3 } & $\begin{array}{c}\text { free energy (Helmholtz } \\
\text { energy) of formation }\end{array}$ & standard enthalpy of formation \\
\hline Mean & 142,0 & 162,6 \\
Variance & $(92,0)^{2}$ & $(94,2)^{2}$ \\
Mode & 123,2 & 124,2 \\
Skewness & 0,139 & 0,140 \\
Excess kurtosis & $-0,577$ & $-0,611$ \\
Pearson $\chi^{2}$ (table estimated) & 0,231 & 0,197 \\
& $\left(\chi_{\text {Table }}^{2}=101,88\right)$ & $\left(\chi^{2}\right.$ Table
\end{tabular}

3) The GMC molecules formation probability calculation with a certain standard enthalpy of formation.

4) The corresponding substances resources assessment (Table 3). 
Table 3. Biologically active compounds resources assessment, calculated from the distribution function for the free energy (Helmholtz energy) of formation

\begin{tabular}{|c|c|c|c|}
\hline \multirow{2}{*}{ № ח/п } & \multirow{2}{*}{ Compound } & \multicolumn{2}{|c|}{ The amount of resources in Sun's mass at T, $K$} \\
\hline & & 100 & 300 \\
\hline 1. & Adenine & $17 \cdot 10^{12}$ & $22 \cdot 10^{12}$ \\
\hline 2. & Guanine & $15 \cdot 10^{12}$ & $20 \cdot 10^{12}$ \\
\hline 3. & Thymin & $16 \cdot 10^{12}$ & $20 \cdot 10^{12}$ \\
\hline 4. & Cytosine & $25 \cdot 10^{12}$ & $31 \cdot 10^{12}$ \\
\hline 5. & Uracyl & $32 \cdot 10^{12}$ & $39 \cdot 10^{12}$ \\
\hline 6. & Glycine & $34 \cdot 10^{12}$ & $41 \cdot 10^{12}$ \\
\hline 7. & Alanine & $18 \cdot 10^{12}$ & $22 \cdot 10^{12}$ \\
\hline 8. & Serine & $16 \cdot 10^{12}$ & $21 \cdot 10^{12}$ \\
\hline 9. & Cysteine & $20 \cdot 10^{12}$ & $27 \cdot 10^{12}$ \\
\hline 10. & Asparaginic acid & $10 \cdot 10^{12}$ & $14 \cdot 10^{12}$ \\
\hline 11. & Asparagine & $6 \cdot 10^{12}$ & $9 \cdot 10^{12}$ \\
\hline 12. & Threonine & $4 \cdot 10^{12}$ & $5 \cdot 10^{12}$ \\
\hline 13. & Proline & $4 \cdot 10^{12}$ & $4 \cdot 10^{12}$ \\
\hline 14. & Glutamine acid & $3 \cdot 10^{12}$ & $4 \cdot 10^{12}$ \\
\hline 15. & Valin & $1 \cdot 10^{12}$ & $1 \cdot 10^{12}$ \\
\hline 16. & Glutamine & $1 \cdot 10^{12}$ & $2 \cdot 10^{12}$ \\
\hline 17. & Histidine & $2 \cdot 10^{12}$ & $2 \cdot 10^{12}$ \\
\hline 18. & Methionine & $1 \cdot 10^{12}$ & $2 \cdot 10^{12}$ \\
\hline 19. & Leucine & $1 \cdot 10^{11}$ & $1 \cdot 10^{11}$ \\
\hline 20. & Isoleucine & $1 \cdot 10^{11}$ & $1 \cdot 10^{11}$ \\
\hline 21. & Phenylalanine & $11 \cdot 10^{7}$ & $3 \cdot 10^{7}$ \\
\hline 22. & Tyrosine & $1 \cdot 10^{11}$ & $1 \cdot 10^{11}$ \\
\hline 23. & Lysin & $3 \cdot 10^{10}$ & $2 \cdot 10^{10}$ \\
\hline 24. & Arginine & $1 \cdot 10^{10}$ & $1 \cdot 10^{10}$ \\
\hline 25. & Tryptophane & $1 \cdot 10^{9}$ & $1 \cdot 10^{9}$ \\
\hline
\end{tabular}

\section{Conclusions}

Data statistical analysis confirms that the distribution of GMO composition is normal, due to the standard enthalpies of formation and free energy (Helmholtz energy). Thus, the criterion $\chi^{2}$ for the sample captured with the volume 67 for standard enthalpies of formation is equal to 0,197 . This is less than the table value of 101,88 (at a confidence level equal to 0,95 ) and for the free energies is equal to 0,231 , which is less than the table value of 101,88 (at a confidence level of 0,95). The skewness of the Pearson's distribution is not big - 0,140 and 0,139 for the standard enthalpies of formation and free energies of formation, respectively (Table 1). Thus, the calculations show that the molecular substance of the universe is a Gaussian super system with considerable chaos in chemical content. Table 2 shows the evaluation of resources of amino acids and nitrogen bases in the 
space, these calculations ared according to the content distribution function of free energy (Helmholtz energy) and the the formation at different temperatures. Resources of important biologically active substances, amino acids and nucleotides were evaluated. These findings indicate the possibility of their formation in the interstellar medium. The results are confirmed by the discovery in the interstellar fullerene medium, etc. (Cami et al., 2010). Thus, the results indicate that significant resources are vital connections in the visible part of the universe and in the existence of life as a natural phenomenon.

\section{References}

Bains, I., Wong, T., Cunningham, M., Sparks, P., Brisbin, D., Calisse, P. ... Burton, M. G. (2006). Molecular line mapping of the giant molecular cloud associated with RCW $106-$ I. ${ }^{13} \mathrm{CO}$. Monthly Notices of the Royal Astronomical Society, 367(4), 1609-1628.

Binney, J, \& Merrifield, M. (1998). Galactic Astronom. USA, Princeton: Princeton University Press.

Cami, J., Bernard-Salas, J., Peeters, E., \& Elizabeth Malek, S. (2010). Detection of C60 and C70 in a Young Planetary Nebula. Science, 329(5996), 1180-1182.

Dolomatov, M. Yu. (2002). Feature of equlibrum thermodynamics systems with chaos of chemical constitutions. International Congres of Chemical Termodinamics. Russia, S. Peterburg.

Dolomatov, M. Yu., \& Kostyleva, E. V. (2001). Characteristics of the organic substances abiogenic synthesis in space natural systems and the problem of oil origin. Oil refining and Petrochemical Industry: The materials of All-Russia Scientific practical Conference. Ufa, pp. 8-14.

Dolomatov, M. Yu. (2004). Features of equilibrium thermodynamics complex systems with chaos of chemical constitutions and allocation of organic matter in the space. Abstracts of International Conference on Complex Systems. USA, Florida, Texas.

Dolomatov, M. Yu. (2005). Fragments of the real substance theory from the hydrocarbon systems to the galaxies. Chemistry. Moskow.

Duval, Ju, Heyer, M., Krawczyk, C., \& Jackson, J. M. (2009). Re-Examining Larson's Scaling Relationships in Galactic Molecular Clouds. The Astrophysical Journal, 699(2), 1092-1103.

Landau, L. D., \& Lifshitz, E. M. (1996). Statistical Physics (3rd Ed. Part), Oxford: Butterworth-Heinemann.

Lee, Y., Snell, R. L., \& Dickman, R. L. (1996). The cold, massive molecular cloud G216-2.5. 2: Structure and kinematics. Astrophysical Journal, 432(1), 167-180.

Lovas, F. (1992). Recommended Rest Frequencies for Observed Interstellar Molecular Microwave Transitions. J. Phys. Chem. Ref, p. 181.

Maloney, P. (1986). Size-density relations in dark clouds: Non-LTE effects. In NASA. Ames Research Center Summer School on Interstellar Processes: Abstracts of Contributed Papers, p 45 - 46 (SEE N87-15043 06-90).

Rudnitskiy, G. (1999). Interstellar molecular clouds. Earth and Universe, 3.

Sakamoto, S. (1996). Physical Conditions of Molecular Gas in the Galaxy. Publications of the Astronomical Society of the Pacific, 106, 1112.

Universität zu Köln. (2012). Molecules in Space. Universität zu Köln. Retrieved from http://www.astro.uni-koeln.de/cdms/molecules

Vasyunin, A. (2008). Development of the theory of numerical simulation of the molecular composition of the interstellar medium. Moscow. 